\title{
Giant Aortic Root Aneurysm Presenting as Acute Type A Aortic Dissection
}

\author{
Guy M. Raz, BS, Sotiris C. Stamou, MD, PhD* \\ Department of Cardiothoracic Surgery, University of lowa Hospitals and Clinics, Carver College of Medicine, lowa City, lowa
}

\begin{abstract}
A 49-year-old woman with four months of increasing episodic palpitations, chest pain, and shortness of breath presented to an outside clinic where a new 4/6 systolic ejection murmur was identified. A transthoracic echocardiogram revealed a large aortic root aneurysm. The patient underwent emergent repair of the dissected root aneurysm with a modified Bentall procedure utilizing a \#19 St Jude Valsalva mechanical valve conduit. Postoperatively, she required a permanent pacemaker placement. Her echo showed ejection fraction improvement from a preoperative $25 \%$ to a postoperative $35 \%$. She was discharged home on postoperative day 7.

Copyright $\odot 2014$ Science International Corp.
\end{abstract}

\section{Key Words}

Aneurysm - Aortic root - Bentall procedure

\section{Case Presentation}

A 49-year-old woman with four months of increasing episodic palpitations, chest pain, and shortness of breath presented to an outside clinic where a new 4/6 systolic ejection murmur was identified. She was then evaluated with a transthoracic echocardiogram which revealed a large aortic root aneurysm. The patient had no family history of connective tissue disease and failed to meet any of the revised Ghent criteria for

Fax +1 2037853346
h-Mail: aorta@scienceinternational.org
http://aorta.scienceinternational.org

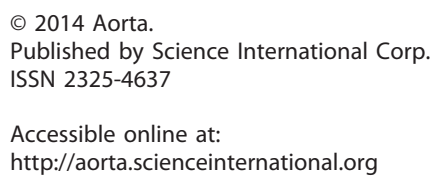

Marfan syndrome. Computed tomography revealed a $9.3 \times 8.9 \times 11.1 \mathrm{~cm}$ aortic root aneurysm with Stanford Type A dissection (Figs. 1 and 2), one of the largest ever reported. The patient underwent emergent repair of the dissected root aneurysm with a modified Bentall procedure utilizing a \#19 St Jude Valsalva mechanical valve conduit. The original trileaflet valve was grossly incompetent due to root dilatation and could not be spared. Pathology showed an intimal tear at the noncoronary sinus of the aortic root. Postoperatively, she required a permanent pacemaker placement. Her echocardiogram showed ejection fraction improvement from a preoperative $25 \%$ to a postoperative $35 \%$. She was discharged home on postoperative day 7 .

\section{Conflict of Interest}

The authors have no conflict of interest relevant to this publication.

\section{Comment on this Article or Ask a Question}

Cite this article as: Raz GM, Stamou SC. Giant Aortic Root Aneurysm Presenting as Acute Type A Aortic Dissection. Aorta 2014; 2(3):121-122. DOI: http://dx.doi.org/10.12945/j.aorta.2014.14-018

*Corresponding author:

Sotiris C. Stamou, MD, PhD

SE 516 General Hospital

University of lowa Hospitals and Clinics

lowa City, IA 52242

Tel: +1 202361 2377, Fax: +1 319356 3891, E-Mail: sotiris-stamou@uiowa.edu 


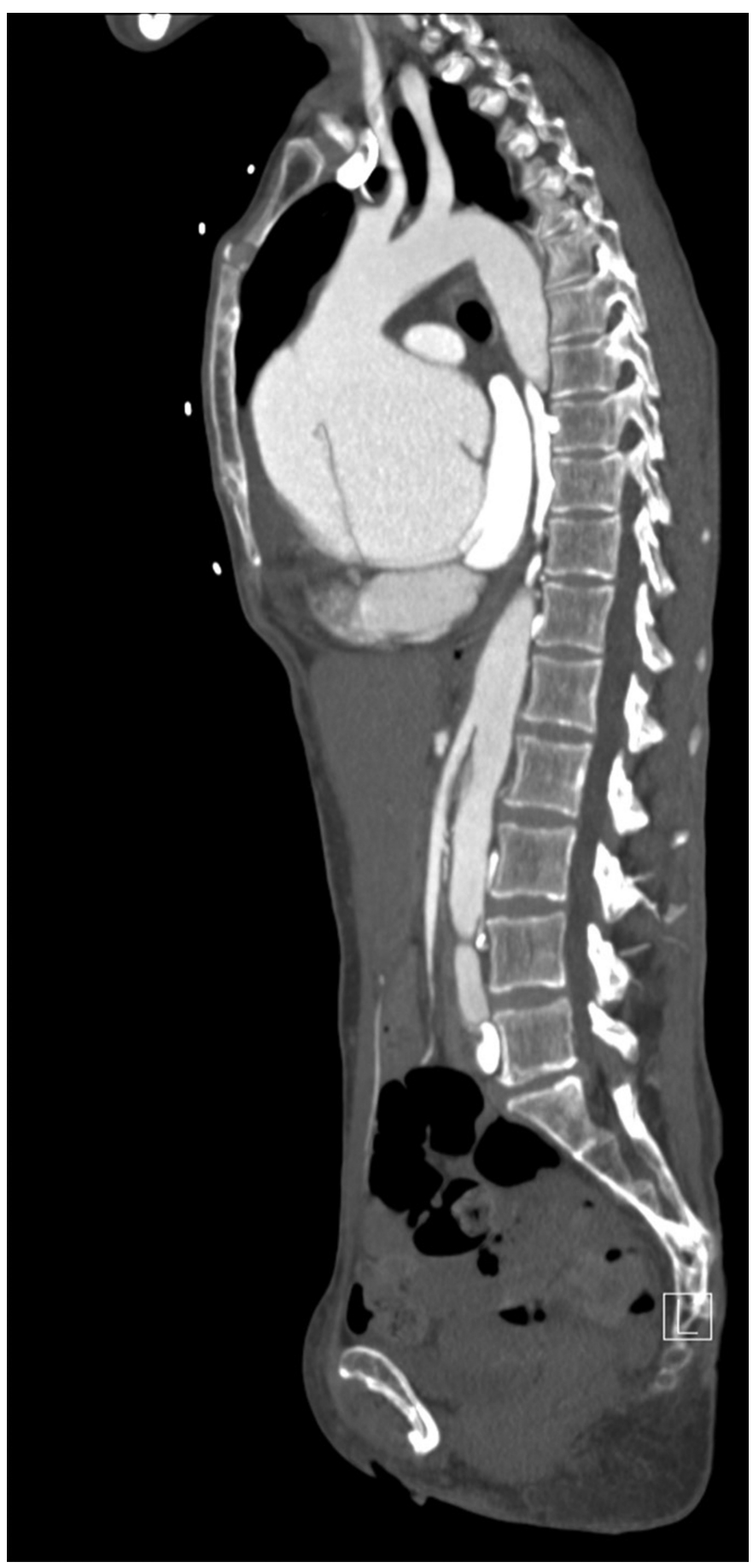

Figure 1. Sagittal computed tomography of chest, abdomen and pelvis demonstrating a giant aortic root aneurysm with Stanford Type A dissection, compressing and displacing the right ventricle.

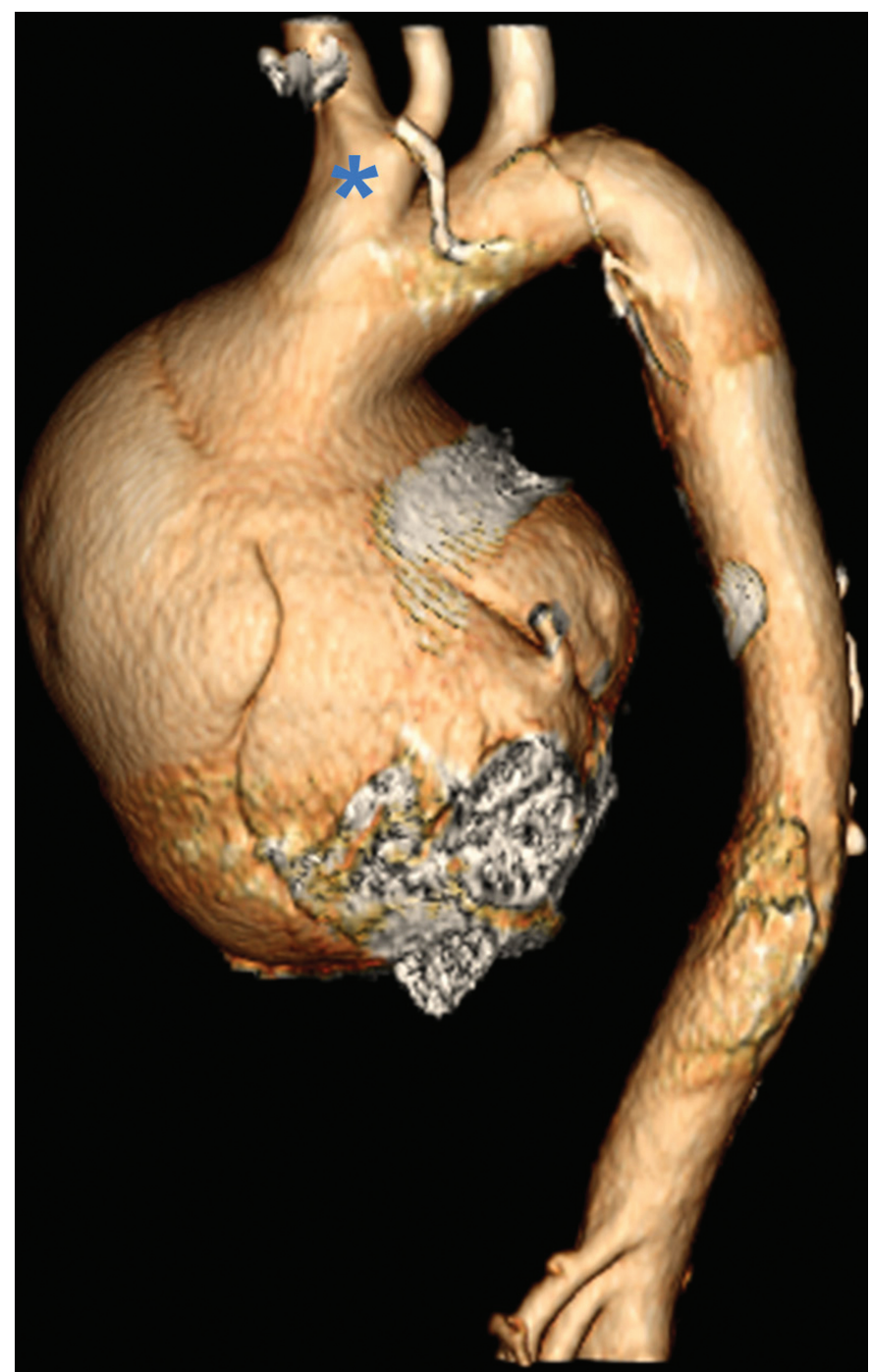

Figure 2. Computed three-dimensional reconstruction of the giant root aneurysm. A bovine arch anomaly is also seen (asterisk). 